\section{Basic Pharmacokinetics}

Jambhekar SS, Breen PJ. Pharmaceutical Press, London, United Kingdom, 2009. Softcover, 416 pages. ISBN 978-085369-772-5. \$59.99.

Basic Pharmacokinetics is a first edition textbook aimed primarily at the entry-level pharmacy student in the United States and Canada. In their foreword, the authors also state that the book would be useful for pharmaceutical scientists, medical students, and graduate students in the pharmaceutical sciences. This textbook covers the following topics: intravascular and extravascular drug administration, single and multicompartmental models, first-order and zero-order pharmacokinetics, noncompartmental pharmacokinetics, drug interactions, biopharmaceutics, and pharmacodynamics.

Each of the 18 chapters includes clear objectives and a comprehensive introduction. The textbook chapters are visually appealing and well written. The authors make good use of equations, diagrams, and graphs to illustrate important pharmacokinetic concepts. The use of problem sets following major sections or chapters, which include detailed work-through answers, makes the textbook very useful as a course reference.

Unfortunately, the book is not as well organized as it could be, both within and between chapters. For example, the single IV bolus one-compartment model is covered in chapter 3, but continuous IV infusion, multiple-dose IV bolus infusion, and intermittent IV infusions are not covered until chapters 10, 11, and 14 , respectively. Important concepts such as area under the concentration-time curve, clearance, and bioavailability are introduced relatively late in the textbook, although the authors do devote an entire chapter to clearance concepts. The organization of the book would make it difficult for someone just starting out in pharmacokinetics to follow from start to finish.

This book devotes 2 chapters to physicochemical factors affecting drug absorption and the role of the dosage form in drug absorption, both of which might otherwise be covered in a pharmaceutics textbook. A better use of space might have been to dedicate chapters to other important pharmacokinetics concepts, such as hepatic clearance (e.g., the well-stirred model) and urinary excretion data, which are covered in less detail elsewhere in the book.

Although primarily a basic pharmacokinetics book, this volume does include many clinical examples. Many of the concepts are first illustrated using a simple nonphysiologic example, followed by a clinical or physiologic example. However, some of the clinical concepts are not covered in enough depth to form a good reference for clinical pharmacokinetics courses, such as those offered in some Canadian undergraduate and graduate pharmacy programs. For example, vancomycin and aminoglycosides are used to illustrate the first-order intermittent IV infusion model; however, the worked-through examples do not include the determination of a new dosing regimen, although they do describe how to calculate volume of distribution and elimination rate constant. The topic of non-steady-state pharmacokinetics is not mentioned at all.

Overall, Basic Pharmacokinetics is well written and accurate, and it clearly states the assumptions for various pharmacokinetic concepts. The layout, which appropriately includes the use of lists, bullets, figures, and tables in addition to the text, makes the individual sections easy to follow. This would be a good textbook for an introductory or basic pharmacokinetics course and may be especially useful for instructors who wish to use certain chapters and problems as homework assignments.

Jennifer Kendrick, BSC, BSc(Pharm), ACPR

PharmD student

Faculty of Pharmaceutical Sciences

University of British Columbia

Vancouver, British Columbia

\section{Textbook of Palliative Medicine}

Bruera E, Higginson IJ, Ripamonti C, von Gunten C, editors. Hodder Arnold Company, London, United Kingdom, 2009. Softcover, 1095 pages. ISBN 978-0-340-96624-2. \$110.00

More than 170 palliative care experts from around the world joined forces in composing this textbook. It is indeed magnificent to have a concentrated pool of experts, from diverse geographic locations, providing a global perspective on the topic of palliative care. Most of the contributors are physicians, with a few representatives from nursing, social work, rehabilitation, epidemiology, and administration. Unfortunately, there is no representation from pharmacy, respiratory therapy, or nutrition.

The book has 16 sections covering a wide spectrum of topics, including the development of palliative medicine, bioethics, pain and other symptoms, emergencies in palliative medicine, and interdisciplinary issues. Each chapter is further divided into short sections. The book has some helpful features, such as a summary of key learning points and a complete list of references at the end of each chapter. For those who would like to further immerse themselves in a certain topic, symbols in the reference section identify seminal primary articles, key review papers, and management guidelines. Evidence scores on 3 levels are also provided: systematic review or meta-analysis, well-designed randomized controlled trial (RCT), and non-RCT or cohort study. Of special interest to pharmacists are the 14 chapters gathered under the topic of pain, with discussions on special populations such as pediatric patients, elderly patients, and patients with challenges related to addiction.

The downside of having numerous writers for a book such as this is that readers must switch among a collage of writing styles, which makes the book challenging to read at times. For 